\title{
Reflets
}

Revue d'intervention sociale et communautaire

\section{Évaluation métrologique de la Mesure de l'offre active de services sociaux et de santé en français en contexte minoritaire}

\author{
Jacinthe Savard, Lynn Casimiro, Josée Benoît et Pier Bouchard
}

Volume 20, numéro 2, automne 2014

L'accès aux services sociaux et de santé en français et la formation des professionnelles et professionnels en situation francophone minoritaire canadienne

URI : https://id.erudit.org/iderudit/1027587ar

DOI : https://doi.org/10.7202/1027587ar

Aller au sommaire du numéro

Éditeur(s)

Reflets, Revue d'intervention sociale et communautaire

ISSN

1203-4576 (imprimé)

1712-8498 (numérique)

Découvrir la revue

Citer cet article

Savard, J., Casimiro, L., Benoît, J. \& Bouchard, P. (2014). Évaluation métrologique de la Mesure de l'offre active de services sociaux et de santé en français en contexte minoritaire. Reflets, 20(2), 83-122.

https://doi.org/10.7202/1027587ar

\section{Résumé de l'article}

Comment peut-on mesurer l'impact des actions entreprises pour améliorer l'offre active de services sociaux et de santé en français dans les communautés francophones en situation minoritaire? Un questionnaire mesurant premièrement les comportements individuels de l'offre active et deuxièmement la perception du soutien organisationnel à faire de l'offre active a été créé. La validité de contenu de la Mesure de l'offre active de services en françaisen contexte minoritaire a été établie à l'aide d'une recension d'écrits, de consultations menées auprès d'expertes et d'experts et d'un sondage Delphi pancanadien. Sa fidélité a été examinée à partir de données recueillies auprès de récents diplômés en santé et en service social. L'outil démontre une bonne consistance interne et des études auprès d'un plus large échantillon sont nécessaires pour augmenter la confiance envers sa stabilité temporelle. Il s'agit des premiers pas d'une démarche visant à mesurer l'évolution des comportements d'offre active à la suite d'activités de formation ou de changements organisationnels en faveur de services en français. Une telle mesure est aussi susceptible d'être utile dans les recherches visant à saisir les déterminants de ces comportements d'offre active.
Tous droits réservés @ Reflets, Revue d’intervention sociale et communautaire, 2014
Ce document est protégé par la loi sur le droit d'auteur. L'utilisation des services d'Érudit (y compris la reproduction) est assujettie à sa politique d'utilisation que vous pouvez consulter en ligne. 


\section{Évaluation métrologique de la Mesure de l'offre active de services sociaux et de santé en français en contexte minoritaire}

Jacinthe Savard, erg. aut. (Ont.), Ph. D., professeure adjointe

École des sciences de la réadaptation et

Clinique universitaire interprofessionnelle en soins de santé primaires

Université d'Ottawa

Lynn Casimiro, PT, Ph. D., directrice de l'enseignement, Hôpital Montfort et

professeure auxiliaire, École des sciences de la réadaptation

Université d'Ottawa

Josée Benoît, Ph. D., associée de recherche

École de service social et École des sciences de la réadaptation

Université d'Ottawa

Pier Bouchard, Ph. D., professeure titulaire

Département d'administration publique

Université de Moncton

Les quatres auteurs sont membres du Groupe de recherche sur la formation professionnelle en santé et en service social en contexte francophone minoritaire (GReFoPS)

Résumé

Comment peut-on mesurer l'impact des actions entreprises pour améliorer l'offre active de services sociaux et de santé en français dans les communautés francophones en situation minoritaire? Un questionnaire mesurant premièrement les comportements 
individuels de l'offre active et deuxièmement la perception du soutien organisationnel à faire de l'offre active a été créé. La validité de contenu de la Mesure de l'offre active de services en français en contexte minoritaire a été établie à l'aide d'une recension d'écrits, de consultations menées auprès d'expertes et d'experts et d'un sondage Delphi pancanadien. Sa fidélité a été examinée à partir de données recueillies auprès de récents diplômés en santé et en service social. L'outil démontre une bonne consistance interne et des études auprès d'un plus large échantillon sont nécessaires pour augmenter la confiance envers sa stabilité temporelle. Il s'agit des premiers pas d'une démarche visant à mesurer l'évolution des comportements d'offre active à la suite d'activités de formation ou de changements organisationnels en faveur de services en français. Une telle mesure est aussi susceptible d'être utile dans les recherches visant à saisir les déterminants de ces comportements d'offre active.

Mots clés : Offre active de services en français, formation des professionnelles ou professionnels en santé et services sociaux, soutien organisationnel, évaluation, minorité, langue et services, qualité des soins et services, sécurité des clientes ou clients/ patientes ou patients

\section{Abstract}

How can we measure the impact of actions taken to improve the active offer of health and social services in French to francophone minority communities? A questionnaire was created to measure firstly individual behaviours of active offer and secondly the perception of organisational support toward it. Content validity of the Active Offer of French Language Services in Minority Context Measure was determined through a literature review, expert consultations and a Canada-wide Delphi survey. Reliability was examined with data collected from recent graduates in health and social services. The questionnaire shows good internal consistency and further studies with a larger sample are necessary to determine its temporal stability. These are the first steps toward measuring the development of active offer behaviours following educational 
or organisational change activities that favour French-language services. It is also likely to help researchers study the determinants of such behaviours.

Key words: Active offer of French services, education of health care and social services professionals, organisational support, evaluation, minority, language and services, quality of care, patient/ client safety

\section{Remerciements}

Ce projet a été rendu possible grâce à une subvention du Consortium national de formation en santé (CNFS) - volet Université d'Ottawa. Les auteures tiennent à remercier Alaâ Zahdarane pour la mise en ligne des questionnaires et Josée Venne pour l'analyse statistique des données, de même que tous les participantes et participants qui ont pris le temps de répondre aux questionnaires.

\section{Introduction}

\section{"Communiquer}

dans une langue qui n'est pas sa langue maternelle peut être particulièrement difficile pour une personne qui doit interagir avec une professionnelle ou un professionnel de la santé. »
Plusieurs études (Evans, Barer et Marmor, 1996; Organisation mondiale de la santé, 2008) montrent l'influence de certains déterminants sociaux, tels l'éducation, le revenu et la position sociale, sur l'état de santé d'une personne. Plus récemment, Bouchard, et collab. (2012) ont démontré que la langue et l'appartenance à un groupe linguistique minoritaire font également partie des déterminants sociaux de la santé au Canada. Communiquer dans une langue qui n'est pas sa langue maternelle peut être particulièrement difficile pour une personne qui doit interagir avec une professionnelle ou un professionnel de la santé. De fait, dans cette interaction, la qualité de la communication est essentielle et ne pas recevoir des soins et des services dans leur langue prédominante peut avoir des conséquences néfastes pour l'adhérence au traitement et pour la sécurité des clientes ou des clients (pour une discussion plus exhaustive sur le sujet, 
"...l'accès à des services sociaux et de santé en français dans les communautés francophones en situation minoritaire devient une priorité pour le gouvernement canadien, non seulement pour se conformer aux droits linguistiques, mais surtout pour assurer la sécurité et la qualité des services sociaux et de santé offerts à toute la population $d u$ pays (Santé Canada, 2007)." voir Drolet, et collab., 2014; Savard, et collab., 2014). C'est ainsi que l'accès à des services sociaux et de santé en français dans les communautés francophones en situation minoritaire devient une priorité pour le gouvernement canadien, non seulement pour se conformer aux droits linguistiques, mais surtout pour assurer la sécurité et la qualité des services sociaux et de santé offerts à toute la population du pays (Santé Canada, 2007). C'est dans cette optique que l'augmentation du nombre et de la disponibilité de personnel pouvant s'exprimer en français devient importante et que le Consortium national de formation en santé (CNFS) a été créé, avec la mission d'assurer la mise en œuvre d'un réseau postsecondaire élargi de formation et de recherche en français pour appuyer les institutions qui offrent, ou pourraient offrir, une formation dans le domaine de la santé dans les communautés francophones en contexte minoritaire (CNFS, 2011).

Par ailleurs, une personne vulnérable peut se sentir intimidée si elle doit revendiquer le respect de ses droits linguistiques; elle peut éprouver une gêne, voire une peur, à demander des services dans sa langue quand les ressources sont déjà limitées (Bouchard, et collab., 2012; 2009; Consortium pour la promotion des communautés en santé, 2011; Drouin et Rivet, 2003).Après plusieurs décennies sans services de santé en français, il peut même se créer chez les personnes en situation linguistique minoritaire une conviction qu'il est impossible d'en recevoir (Société santé en français, 2007). Le concept de l'offre active devient donc un outil essentiel pour accroître l'accessibilité aux services en français. Selon Bouchard, et collab. (2012, p. 46), «l'offre active peut être considérée comme une invitation, verbale ou écrite, à s'exprimer dans la langue officielle de son choix. L'offre de parler dans sa langue officielle de son choix doit précéder la demande de services".

Dans le contexte du présent projet, le concept d'offre active se concrétise par les éléments suivants : 1. les services en français sont visibles, disponibles et facilement accessibles à la clientèle; 2. le personnel informe les clientes ou clients qu'ils peuvent s'adresser à eux et se faire servir dans les deux langues officielles; 3. les particularités linguistiques et culturelles de chaque client sont prises en compte; 4 . le personnel et les organisations 
"...offrir activement des services en français dépasse le simple affichage d'offre de services bilingues. Il s'agit de poser des actions adaptées à la culture linguistique minoritaire pour que les francophones se sentent à l'aise avec les services offerts dans leur langue. " appliquent cette approche dans tout le continuum de soin (accueil, prise en charge, intervention, soutien et aiguillage), sans que la responsabilité incombe seulement au client d'en faire la demande. L'offre active permet aux clientes et clients de se sentir à l'aise d'utiliser la langue officielle de leur choix lorsqu'ils reçoivent des services. De plus, le grand public est convaincu que s'il utilise la langue officielle de son choix, il recevra des services et des soins de qualité.

Ainsi, offrir activement des services en français dépasse le simple affichage d'offre de services bilingues. Il s'agit de poser des actions adaptées à la culture linguistique minoritaire pour que les francophones se sentent à l'aise avec les services offerts dans leur langue. Un exemple d'action possible de la part du personnel consiste à adresser aussi en français les messages d'accueil pour démontrer qu'il est lui-même ouvert à s'exprimer dans cette langue (Comité des services en français de Fredericton, 2006). Un autre exemple est de proposer de la documentation imprimée conjointement dans les deux langues afin que la personne qui est plus à l'aise en français dans certains sujets et en anglais dans d'autres - ou la personne francophone dont l'aidant principal est plus à l'aise en anglais - plutôt que de devoir choisir l'une ou l'autre version, puisse avoir accès aux deux.

Si l'importance de recevoir des services dans sa langue est déjà bien documentée, les écrits scientifiques sur le concept d'offre active et les facteurs expliquant la mise en place de services dans les deux langues officielles sont plus rares, tant dans le domaine de la santé (Forgues, et collab., 2011) que dans d'autres domaines, comme celui de la justice (Cardinal et Sauvé, 2010). De plus, ce concept est parfois confondu avec le bilinguisme (Vézina, et collab., 2014), ce qui laisse à penser que des études sur les pratiques d'offre active seront d'une grande utilité.

\section{Les défis de l'offre active de services en français}

Outre le manque de personnel en santé et en services sociaux pouvant s'exprimer en français, il appert que même le personnel 
francophone ou bilingue rencontre des obstacles au moment de mettre en pratique des mécanismes d'offre active de services en français (Bouchard et Vézina, 2009). Parmi ces obstacles, on retrouve la difficulté de maintenir sa compétence linguistique en français lorsque la langue de travail est l'anglais (Bouchard et Vézina, 2009); la nécessité de composer avec une clientèle diversifiée quant à la langue et à la culture; le besoin de traduire ou d'adapter des ressources puisqu'il existe peu d'outils d'évaluation, de traitement, d'information et d'éducation en français; le fait d'être souvent sollicité par des collègues pour effectuer de la traduction ou agir en tant qu'interprète, ce qui peut mener à une surcharge de travail (Bouchard et Vézina, 2009; Drolet, et collab., 2014); et le manque formation sur les particularités des communautés francophones en contexte minoritaire, sur les enjeux liés à l'offre active et sur les gestes à poser afin d'améliorer la situation (Benoit, et collab., 2014; Bouchard et Vézina, 2009).

La responsabilisation et le leadership des gestionnaires sont souhaités afin d'instaurer des orientations claires sur les services en français et de fournir au personnel des outils nécessaires à leur mise en pratique (Bouchard etVézina, 2009; Bouchard, et collab., 2012; Charland, 2011). Ce soutien organisationnel n'est toutefois pas toujours présent pour faciliter la prestation de services en français par le personnel francophone ou bilingue. Même dans les situations où une intervenante ou un intervenant bilingue a été embauché pour ses compétences professionnelles et linguistiques, il arrive que les gestionnaires n'en fassent que peu d'usage et qu'ils exigent, par exemple, que les différentes communications entre les membres du personnel se fassent en anglais afin d'éviter l'exclusion d'une intervenante ou d'un intervenant unilingue anglophone (Bouchard et Vézina, 2009).

Il semble donc qu'une meilleure préparation du personnel à mettre en pratique les mécanismes d'offre active serait nécessaire, tout comme une meilleure sensibilisation des gestionnaires. De fait, la valorisation et l'outillage des ressources humaines pour la qualité et la sécurité des services en français figurent parmi les priorités du plan d'action de la Société santé en français pour 2013-2018 afin d'améliorer l'accès aux services de santé en français et l'état 
de santé des francophones au Canada (Santé société en français, 2012). Considérant les efforts investis dans l'amélioration de l'offre active de services en français (CNFS, 2012; Dubouloz, et collab., 2014 [accepté]; Santé société en français, 2012), il est entendu qu'il faudra évaluer les effets de ces activités. Dans l'élaboration de programmes de formation, le CNFS et ses institutions membres et partenaires communautaires ont besoin de tels outils pour évaluer les interventions qui stimulent l'engagement des étudiantes et des étudiants et celui des intervenantes et des intervenants envers la communauté francophone.

Tenant compte des connaissances entourant l'offre active de services en français, des défis qui y sont associés et des efforts actuels pour offrir de la formation dans le domaine, notre équipe, constituée de membres du Groupe de recherche sur la formation professionnelle en santé et en service social en contexte francophone minoritaire (GReFoPS), s'est interrogée sur les façons de mesurer l'offre active.

\section{Mesurer l'offre active}

Comment peut-on mesurer l'impact des actions entreprises pour améliorer l'offre active de services sociaux et de santé en français dans les communautés francophones en situation minoritaire? Comment peut-on en étudier les déterminants, c'est-à-dire les facteurs qui favorisent la mise en pratique de ses mécanismes? Il

"Les consultations et la recension des écrits ont révélé l'existence d'aucun outil permettant de quantifier les comportements d'offre active observés chez les intervenantes ou intervenants en santé et en services sociaux." y aurait lieu de pouvoir mesurer l'offre active pour identifier les situations où elle est plus présente. Les consultations et la recension des écrits ont révélé l'existence d'aucun outil permettant de quantifier les comportements d'offre active observés chez les intervenantes ou intervenants en santé et en services sociaux. Toutefois, une vingtaine de documents clés décrivaient des exemples d'actions ou de politiques entreprises par les établissements locaux ou les gouvernements provinciaux pour la favoriser dans les services sous leur juridiction, ainsi que quelques grilles d'évaluation à l'intention des milieux qui souhaitent améliorer leur offre de services en français (Charland, 2011; Comité santé en français de Fredericton, 2006; Gouvernement de l'Île-du-Prince-Édouard, 2011; Gouvernement du Manitoba, 
2011; Réseau de santé en français de l'est de l'Ontario (2010); Société Santé en français, 2010; Société Santé et mieux-être en français du Nouveau-Brunswick (s. d.).

Ce constat a conduit les auteurs du présent article à mettre au point la Mesure de l'offre active de services en français en contexte minoritaire suivant un processus adapté de celui proposé par Bradburn, Sudman et Wansink (2004), où les items du questionnaire sont générés à partir de ce qui est retrouvé dans les écrits et à la suite de consultations menées auprès d'expertes et d'experts. En s'inspirant des documents clés sur l'offre active de services en français, notamment de pratiques innovatrices en la matière, il a été possible d'extraire diverses actions organisationnelles qui pouvaient être transposées à l'action individuelle pour établir une première liste de comportements observables chez les personnes qui s'y adonnent. La consultation menée auprès d'expertes et d'experts au sujet de cette liste de comportements individuels a fait ressortir que les personnes qui travaillent dans un milieu où un nombre important d'actions sont réalisées par les employeurs ou les établissements pour démontrer une volonté d'offre active de services en français seraient plus portées à faire des efforts pour offrir activement ces services et qu'il serait important de mesurer simultanément ces indicateurs de soutien organisationnel à l'offre active. Il en est résulté la création d'un questionnaire en deux sections, l'une portant sur les comportements individuels d'offre active chez les intervenantes ou intervenants et l'autre sur leur perception du soutien organisationnel face à cette dernière.

La validité de contenu de cet outil a été examinée au moyen d'un sondage Delphi pancanadien. La démarche, décrite plus en détail dans Savard, et collab. (2014 [accepté]), a conduit à la création de la version expérimentale du questionnaire dont l'évaluation métrologique est rapportée dans le présent article.

\section{L'évaluation métrologique d'un outil de mesure}

Le processus d'évaluation métrologique d'un outil de mesure s'effectue généralement en plusieurs étapes. La validité de contenu 
par un groupe d'expertes et d'experts est un préalable important à l'acceptation d'une mesure. Toutefois, le jugement des pairs n'est pas suffisant et des preuves empiriques doivent démontrer que l'outil mesure bien ce qu'il prétend mesurer (la validité). De plus, avant de juger de la validité d'un outil, il importe de vérifier s'il démontre une reproductibilité dans les réponses obtenues (la fidélité) (Streiner et Norman, 2008).

Bradburn, et collab. (2004) suggèrent d'effectuer d'abord un test pilote auprès d'un petit échantillon (entre 20 et 50 personnes) représentatif de la population auprès de laquelle la mesure sera utilisée. Ce premier test permet d'éliminer les questions qui n'apportent aucune information discriminante ou qui ne paraissent pas apporter le type d'information recherchée et de réviser les questions qui ont causé des difficultés aux répondantes et répondants (par exemple, celles qui ont de faibles taux de réponse). Il permet aussi une première évaluation métrologique de l'outil. Puis, Bradburn, et collab suggèrent d'effectuer un nouveau test pilote si les révisions sont substantielles, et de soumettre la nouvelle version du questionnaire à un échantillon plus important pour confirmer ses propriétés métrologiques.

Parmi les propriétés métrologiques d'un outil d'évaluation, la fidélité représente la reproductibilité de la mesure, c'est-àdire la probabilité que la mesure donne des résultats similaires à différentes occasions ou lorsque mesurée de différentes manières. Un coefficient de fidélité représente la proportion de la variabilité des résultats qui est due à la vraie différence entre les personnes (Streiner et Norman, 2008). La consistance interne est une forme de fidélité qui représente l'homogénéité ou le degré de constance des réponses aux divers items ou énoncés d'une mesure. Elle est fondée sur l'hypothèse que si un questionnaire comporte plusieurs items qui mesurent la même dimension, il devrait y avoir une forte corrélation entre chacun des énoncés. Une consistance interne élevée est nécessaire pour chacune des échelles pour lesquelles on souhaite calculer un score total afin que ces résultats signifient réellement une plus grande quantité des dimensions évaluées (Gardner, 1995; Sylverstein, et collab., 1992). Une autre forme importante de fidélité est la stabilité 
temporelle des résultats (ou fidélité test-retest). La stabilité temporelle est la corrélation entre deux passations de l'outil d'évaluation, lorsqu'aucun changement n'est survenu entre les deux passations par le même sujet. Cette propriété, observée en l'absence d'intervention, augmente la confiance qu'un changement observé dans les résultats à la suite d'une intervention soit réellement dû à l'intervention.

La validité est la capacité d'un outil à bien refléter le concept que l'on veut mesurer. Elle est une question de degré plus qu'une propriété fixe. C'est l'accumulation de résultats de recherches qui informent sur la validité d'un outil d'évaluation. La validité de contenu se rapporte au choix des items d'un outil d'évaluation. Le contenu doit être représentatif de toutes les facettes du phénomène à l'étude. De plus, il ne doit pas contenir d'éléments qui ne sont pas reliés à ce phénomène. La validité de construit vise à confirmer le cadre théorique sous-jacent à la mesure et à vérifier les hypothèses quant aux liens entre les indicateurs retenus et le phénomène que l'on cherche à mesurer (Tremblay, et collab., 2004).

Le but de la présente recherche était d'effectuer une première évaluation empirique de la Mesure de l'offre active de services en français en contexte minoritaire et d'améliorer l'outil à partir des résultats obtenus. Les objectifs spécifiques étaient les suivants :

- Déterminer la fidélité de la version expérimentale de l'outil, en particulier sa consistance interne et sa stabilité temporelle;

- Réviser l'outil (création de la version 1.0) en éliminant ou modifiant les énoncés qui semblaient problématiques en fonction de trois critères : le taux de non-réponse, la consistance interne et la stabilité temporelle;

- Déterminer la fidélité de la nouvelle version 1.0, en particulier sa consistance interne et sa stabilité temporelle;

- Amorcer l'examen de la validité de construit de l'outil (version 1.0) en examinant les liens entre les comportements individuels d'offre active et le soutien organisationnel perçu en lien avec l'offre active. 


\section{Méthode}

Il s'agit d'une étude de type métrologique qui était incluse dans une étude plus large des déterminants possibles de l'offre active de services en français. L'étude a reçu l'approbation du comité de déontologie de la recherche de l'Université d'Ottawa.

\section{Population à l'étude et échantillon}

L'ensemble des professionnelles ou professionnels de la santé et des services sociaux capables d'offrir des services en français et travaillant dans des milieux où le français n'est pas la langue principale représentait la population cible pour cette étude. L'échantillon étudié était constitué d'un sous-ensemble de cette population, soit les diplômées et diplômés des cinq dernières années des programmes de formation en santé et en service social des universités d'Ottawa et de Moncton. Il s'agissait d'un échantillon de convenance pouvant facilement être recruté par les bureaux des anciens des deux universités participantes. Par ailleurs, la période postdiplôme de cinq ans représentait un seuil jugé approprié afin que les répondantes et répondants puissent participer à l'étude plus large portant sur les déterminants de l'offre active qui nécessitait des expériences de formation et de travail rapprochées.

Les critères d'inclusion précis étaient de détenir un diplôme d'un programme CNFS de l'Université d'Ottawa ou de l'Université de Moncton depuis cinq ans ou moins; travailler ou avoir travaillé dans une province autre que le Québec; y avoir occupé un emploi lié à la santé ou aux services sociaux.

\section{Collecte de données}

La collecte de donnée comportait un questionnaire de données sociodémographiques, la Mesure de l'offre active de services en français en contexte minoritaire et un questionnaire des déterminants possibles de l'offre active. Ce dernier questionnaire était inclus pour les besoins de l'étude plus large. Les questionnaires ont été 
"La Mesure de l'offre active de services en français en contexte minoritaire est un questionnaire d'autoévaluation où la personne cote ellemême la fréquence de ses comportements d'offre active et sa perception de la présence de soutien organisationnel à cette dernière dans son milieu de travail.» mis en ligne à l'aide du logiciel FluidSurveys, pour la période allant du 5 décembre 2012 au 21 janvier 2013. Au total, on a estimé que répondre aux trois questionnaires prendrait de 45 à 60 minutes.

Une invitation a été envoyée par courriel aux diplômés des cinq dernières années des programmes de formation ciblés par le CNFS des universités d'Ottawa $(\mathrm{n}=986)$ et de Moncton $(\mathrm{n}=1010)$ par le bureau des anciens de ces universités, accompagnée du lien Internet pour accéder aux questionnaires. Toutefois, à l'Université d'Ottawa, lorsque le programme est disponible dans les deux langues officielles, les dossiers du bureau des anciens ne contenaient pas nécessairement la langue des études. On a fait parvenir le lien aux diplômés des programmes ciblés qui avaient indiqué le français comme langue préférée de communication avec l'université, ce qui peut être différent de la langue des études. Un courriel de rappel a aussi été envoyé à toutes ces personnes au début du mois de janvier 2013. De plus, afin d'augmenter la participation, l'étude a été annoncée dans les médias sociaux suivants : la page Facebook de l'équipe de recherche, celle du CNFS, le bulletin hebdomadaire du Réseau des services de santé en français de l'Est de l'Ontario (Réseau Express) et le bulletin du Regroupement des intervenantes et des intervenants de la santé et des services sociaux de l'Ontario (RIFSSSO).

Afin de procéder à l'étude de la stabilité temporelle de l'outil, à la fin des questionnaires, on demandait aux participantes et aux participants s'ils accepteraient d'y répondre une seconde fois et, dans l'affirmative, d'indiquer leur adresse courriel. Les 26 personnes qui ont répondu positivement ont reçu une deuxième invitation par courriel entre le 15 février et le $1^{\text {er }}$ mars 2013, comportant un lien les dirigeant uniquement vers la Mesure de l'offre active de services en français en contexte minoritaire.

\section{Mesure de l'offre active de services en français en contexte minoritaire}

La Mesure de l'offre active de services en français en contexte minoritaire est un questionnaire d'autoévaluation où la personne cote ellemême la fréquence de ses comportements d'offre active et sa 
perception de la présence de soutien organisationnel à cette dernière dans son milieu de travail. La version expérimentale de la mesure utilisée dans cette recherche était constituée d'une première section portant sur les comportements individuels d'offre active qui comportait 27 énoncés et 3 questions ouvertes, regroupés en 3 dimensions ou sous-échelles, et d'une deuxième section portant sur la perception du soutien organisationnel qui contenait 42 énoncés regroupés en 5 dimensions ou sous-échelles. Chaque énoncé évaluait la fréquence du comportement décrit sur l'échelle allant de 1 (jamais) à 4 (toujours). Les répondantes et répondants pouvaient aussi indiquer que le comportement ne s'appliquait pas à leur situation. Les questions ouvertes leur permettaient d'indiquer s'ils utilisaient d'autres mécanismes d'offre active. Enfin, pour détecter les biais de réponses systématiques, la version expérimentale de l'outil comportait quatre énoncés formulés négativement : répéter souvent le comportement en question indiquait une absence d'offre active. De plus, deux questions étaient cotées sur une échelle différente : la première sur la fréquence à laquelle des formations sont offertes en français et la seconde sur les obstacles perçus à l'offre de services en français.

\section{Analyse}

\section{Distribution des réponses et calcul d'un score total pour chaque sous-échelle}

Dans un premier temps, la distribution des réponses de chaque énoncé de la Mesure de l'offre active de services en français en contexte minoritaire a été examinée à l'aide de statistiques descriptives. Une variabilité dans les réponses est souhaitée pour que l'instrument soit discriminant. Les faibles taux de réponse peuvent dénoter une difficulté quant à la formulation d'un énoncé ou un manque de pertinence chez ce dernier. Ainsi, les énoncés ayant obtenu un taux de réponse inférieur à $25 \%$ ont été examinés. Pour cette analyse, le choix de réponse "ne s'applique pas" a été considéré comme une absence de réponse. Dans le calcul d'un score total, il était difficile de déterminer si 
la réponse "ne s'applique pas " reflétait une situation clinique où le comportement ne serait jamais approprié ou s'il indiquait une absence du comportement pouvant découler d'un manque d'offre active. Par exemple, pour l'énoncé «Je veille à ce qu'il y ait des documents informatifs et des outils de divertissement en français (p. ex., dépliants, revues, journaux, radio, télévision, jeux) dans les aires communes (salle d'attente ou autre) », la réponse «ne s'applique pas » peut refléter deux choses différentes : 1.le fait d'avoir un environnement de travail où il n'y a pas d'aire commune ne constitue pas un manque d'offre active ou 2. la répondante ou le répondant ne croit pas que ce soit sa responsabilité de vérifier ce que contiennent les aires communes, reflétant ainsi un moins grand comportement d'offre active. Deux scores totaux ont donc été calculés pour chaque échelle ou sous-échelle : un score brut attribuant une note de " 0 " à chaque réponse " ne s'applique pas " ou à chaque réponse manquante et un score pondéré, qui ne considérait que les énoncés notés par la répondante ou le répondant.

Enfin, les réponses aux questions ouvertes ont été examinées pour vérifier la présence de comportements d'offre active qui ne seraient pas inclus dans les énoncés du questionnaire.

\section{Consistance interne de la version expérimentale}

La consistance interne du questionnaire a été évaluée à l'aide du coefficient alpha de Cronbach $(\alpha)$. Un coefficient entre 0,7 et 0,9 est recommandé par Streiner et Norman (2008), alors qu'un résultat supérieur à 0,9 pourrait indiquer la présence d'un grand nombre d'items redondants. Le coefficient $\alpha$ total pour l'ensemble des énoncés de chaque sous-échelle a été calculé. Il a ensuite été recalculé en retirant un énoncé à la fois afin de vérifier si la consistance interne de la sous-échelle s'améliorait. Lorsque c'était le cas, les énoncés contribuant à une diminution de la cohérence interne de leur sous-échelle ont été examinés pour déterminer s'ils étaient plus en lien avec une autre sous-échelle ou s'ils devaient simplement être retirés du questionnaire. 


\section{Stabilité temporelle de la version expérimentale}

La stabilité temporelle du questionnaire expérimental a d'abord été examinée par la comparaison des scores moyens pour chacune des deux passations puis à l'aide de deux coefficients, soit le coefficient kappa pour la fidélité de chaque énoncé individuel et le coefficient de corrélation intraclasse (CCI modèle 3,1 - modèle à effets mixtes à deux facteurs, cohérence absolue, mesures uniques) pour la fidélité des résultats totaux de chaque échelle. Ce modèle est approprié lorsque des sujets s'autoévaluent à deux occasions (Schuck, 2004). Les énoncés qui ont obtenu un coefficient kappa inférieur à 0,20 (pauvre selon l'échelle de Landis et Koch, 1977) ont été examinés afin de déterminer s'ils devaient être modifiés ou éliminés du questionnaire. Pour les scores totaux, un CCI de moins de 0,60 est jugé insuffisant, de 0,60 à 0,70 suffisant et de plus de 0,70 bon, lorsque les résultats sont utilisés pour comparer des groupes dans un contexte de recherche (Nunnally et Bernstein, 1994).

\section{Révision de l'outil : création de la version 1.0}

Les énoncés qui semblaient problématiques sur ces trois critères (fort taux de "ne s'applique pas ", faible stabilité temporelle et ne contribuant pas à la consistance interne de leur échelle) ont été examinés par les trois premières auteures. La décision de conserver ou de rejeter un énoncé reposait sur la grandeur de son effet sur la consistance interne $(\alpha)$ et la fidélité (CCI) de chaque souséchelle, tout en retenant un nombre suffisant d'énoncés pour bien représenter les diverses facettes de l'offre active.

\section{Évaluation de la version 1.0 : fidélité et validité de construit}

À la suite de cette démarche, la consistance interne $(\alpha)$ et la stabilité temporelle (CCI) de la nouvelle version de l'outil ont été réexaminées. L'examen de la validité de construit de l'outil a été amorcé en calculant les corrélations entre le score de chaque sous-échelle et le score total de son échelle. Si chaque échelle mesure une dimension distincte de l'offre active, on s'attendra 
à des corrélations de faibles à modérées entre les sous-échelles et à des corrélations fortes entre chaque sous-échelle et le score total. Les corrélations entre les comportements individuels d'offre active et le soutien organisationnel perçu ont aussi été étudiées. De l'avis du groupe d'expertes et d'experts ayant participé à la validité de contenu de l'outil, le soutien organisationnel à faire de l'offre active devrait être un prédicteur important des comportements individuels d'offre active. On s'attend donc à une corrélation positive modérée entre les deux questionnaires, puisqu'ils mesurent des concepts apparentés, mais distincts. Toutes ces corrélations ont été mesurées à l'aide des coefficients de corrélation de Pearson.

\section{Résultats}

\section{Caractéristiques des participantes et participants}

Comme illustré à la Figure 1, après avoir éliminé certains répondantes ou répondants qui n'avaient jamais travaillé dans le domaine des services sociaux et de la santé ou à l'extérieur du Québec, l'échantillon final pour cette étude était composé de 60 participantes et participants. Parmi eux, un sous-groupe de 22 personnes (sur les 26 qui avaient accepté qu'on les sollicite une $2^{\mathrm{e}}$ fois) a rempli la Mesure de l'offre active de services en français en contexte minoritaire à deux reprises pour l'analyse de sa stabilité temporelle.

Le Tableau 1 présente les principales caractéristiques des participantes et des participants. On peut voir que ce sont majoritairement des personnes dont la langue maternelle est le français qui ont répondu au questionnaire. Provenant principalement de l'Ontario et du Nouveau-Brunswick, elles exerçaient diverses professions (Tableau 1) et représentaient un large éventail de milieux de pratique (non illustré). Un petit nombre $(13 \%)$ travaillait au Québec au moment de l'étude, mais pouvait se référer à une expérience de travail dans une autre province (Tableau 2). Parmi les personnes qui répondaient pour une expérience en Ontario, 45 (75\% de l'échantillon total) 
étaient de la région d'Ottawa et des environs. Comme on peut le constater au Tableau 3, la majorité des participantes et des participants $(83,3 \%)$ travaillaient dans des milieux offrant des services dans les deux langues officielles. De plus, $40 \%$ d'entre eux ont indiqué offrir des services en français à plus de $60 \%$ de leur clientèle. Pour un autre $30 \%$ de participantes ou participants, les francophones constituaient environ la moitié de la clientèle.

Figure 1 - Constitution de l'échantillon

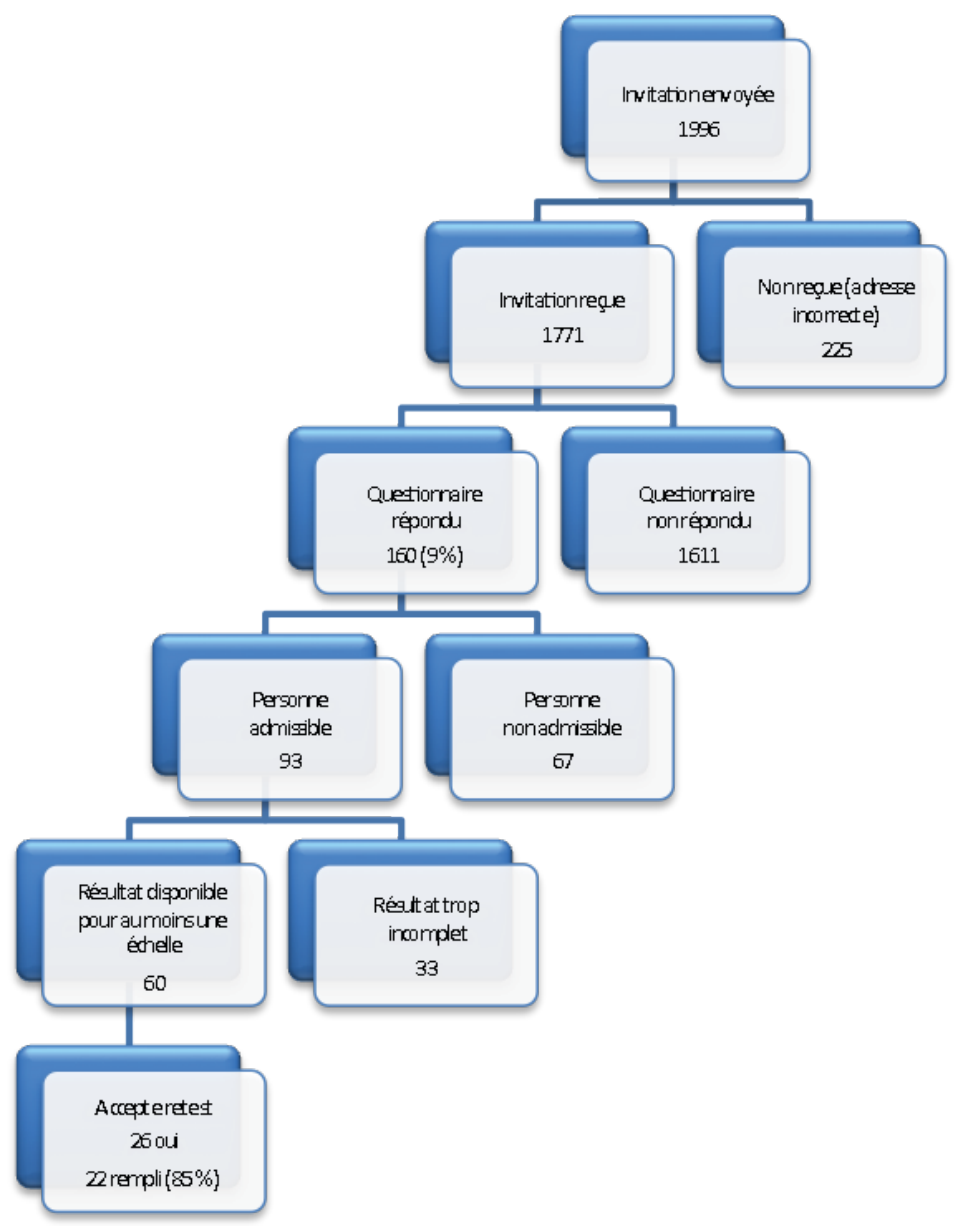


Tableau 1 - Caractéristiques des participantes et des participants

\begin{tabular}{lr}
\hline & $\mathbf{n}(\%)$ \\
\hline Langue maternelle & \\
- Français & $41(68,3 \%)$ \\
- Français et anglais & $1(1,7 \%)$ \\
- Anglais & $4(6,7 \%)$ \\
- Autres & $3(5,0 \%)$ \\
- Données manquantes & $11(18,3 \%)$ \\
\hline Statut d'emploi & \\
- Travail à temps plein & \\
- Travail à temps partiel & $48(80,0 \%)$ \\
- Aux études & $3(5,0 \%)$ \\
- Autres & $6(10,0 \%)$ \\
& - Études et travail à temps partiel \\
- Résidente en médecine familiale & $2(3,3 \%)$ \\
Domaine du diplôme d'études & $1(1,7 \%)$ \\
- Audiologie & \\
- Ergothérapie & $2(3,3 \%)$ \\
- Kinésiologie, activité physique & $3(5,0 \%)$ \\
- Médecine & $2(3,3 \%)$ \\
- Nutrition & $10(16,7 \%)$ \\
- Orthophonie & $4(6,7 \%)$ \\
- Service social & $4(6,7 \%)$ \\
- Soins infirmiers & $3(5,0 \%)$ \\
- Autres & $15(25,0 \%)$ \\
- Donnée manquante & $10(16,7 \%)$ \\
\hline
\end{tabular}

Tableau $2-$ Provenance des participantes et participants

\begin{tabular}{lccc}
\hline & $\begin{array}{c}\text { Lieu de résidence } \\
\mathbf{n}(\%)\end{array}$ & $\begin{array}{c}\text { Lieu de travail } \\
\text { actuel } \\
\mathbf{n}(\%)\end{array}$ & $\begin{array}{c}\text { Milieu de travail } \\
\text { pour les réponses* } \\
\mathbf{n}(\%)\end{array}$ \\
\hline - Ontario & $38(63,3 \%)$ & $42(70,0 \%)$ & $49(81,7 \%)$ \\
- Nouveau-Brunswick & $9(15,0 \%)$ & $9(15,0 \%)$ & $10(16,7 \%)$ \\
- Colombie-Britannique & $1(1,7 \%)$ & $1(1,7 \%)$ & $1(1,7 \%)$ \\
- Québec & $12(20,0 \%)$ & $8(13,3 \%)$ & \\
\hline Total & $\mathbf{6 0}$ & $\mathbf{6 0}$ & $\mathbf{6 0}$ \\
\hline
\end{tabular}

* après répartition des participantes et participants qui travaillent au Québec en fonction de la province où ils ont déjà travaillé et à laquelle ils se réferent pour répondre au présent questionnaire. 
Tableau 3 - Caractéristiques linguistiques du milieu de travail

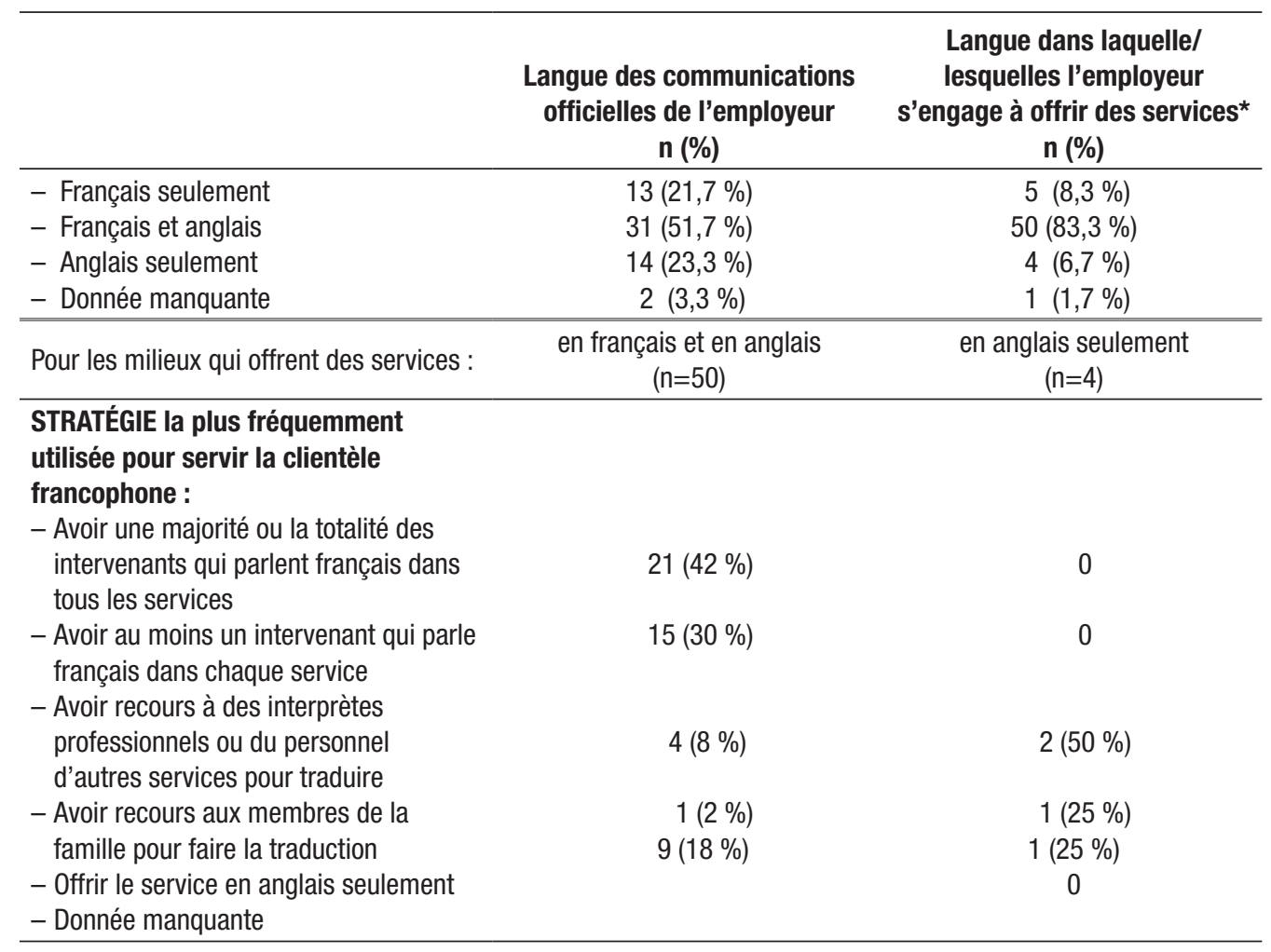

* la question était posée aux répondantes ou répondants, sans préciser si le terme s'engager signifiait une obligation légale ou une volonté de l'employeur.

\section{Évaluation de la version expérimentale de l'outil}

L'analyse de la distribution des réponses révèle chez elles une bonne variabilité : aucun énoncé n'a obtenu une réponse unique et il n'y a que quatre énoncés pour lesquels seuls 2 des 4 choix ("souvent » et " toujours ») ont été sélectionnés. L'analyse des scores totaux et de leurs écarts-types, pour les deux méthodes de calcul (scores bruts et scores pondérés), démontre aussi une 
bonne variabilité des réponses. L'analyse a aussi révélé que 18 des énoncés de la version expérimentale de l'outil ont obtenu des taux de non-réponse supérieurs à $25 \%$ et 14 énoncés ont démontré une pauvre stabilité temporelle (coefficient kappa $<0,20$ ). Ces faibles stabilités temporelles avaient un impact sur deux souséchelles, Comportements : accueil et prise en charge et Comportements : intervention, pour lesquelles les coefficients de corrélation intraclasse étaient inférieurs à 0,60 . L'étude des scores bruts et pondérés de chacune des passations ne révèle pas de différences statistiquement significatives entre les scores de la première passation et ceux de la seconde, sauf dans le cas de la sous-échelle Comportements : accueil et prise en charge où les résultats de la seconde passation étaient plus faibles. Ce biais systématique semble attribuable au fait que les participantes et participants ont été plus critiques lorsqu'ils répondaient à certaines questions une seconde fois. Plusieurs énoncés, qui étaient jugés comme n'étant pas applicables dans leur contexte lors de la première passation, obtenaient des réponses à la seconde passation, indiquant dans la plupart des cas une faible occurrence du comportement.

De plus, la consistance interne de deux sous-échelles, Soutien organisationnel : soutien et aiguillage et Soutien organisationnel : gestion et gouvernance, n'était pas satisfaisante (coefficient $\alpha<0,70$ ). L'étude démontrait que 11 énoncés ne contribuaient pas bien à la souséchelle où ils avaient été placés puisque les retirer de la sous-échelle faisait augmenter son coefficient $\alpha$. Enfin, l'utilisation de choix de réponses différents pour deux groupes d'énoncés, « formation en français " et "obstacles ", a créé des difficultés pour le calcul de scores totaux par sous-échelle et l'impossibilité d'inclure ces énoncés dans le calcul de la consistance interne.

Les réponses aux questions ouvertes ont fourni des précisions sur la façon d'effectuer certains comportements d'offre active inclus dans le questionnaire, mais n'ajoutaient pas de nouveaux comportements qui auraient pu suggérer l'ajout de nouveaux énoncés. 


\section{Création de la Mesure de l'offre active de services en} français en contexte minoritaire, version 1.0 (voir Annexe).

Tableau 4 - Modifications apportées au questionnaire

\begin{tabular}{|c|c|}
\hline Version expérimentale & Version 1.0 \\
\hline Comportements d'offre active & Comportements d'offre active \\
\hline $\begin{array}{l}\text { Accueil et prise en charge } \\
\text { Quelles sont les actions que je prends personnellement } \\
\text { pour faire connaître le fait que je peux offrir des services en } \\
\text { français? } \\
\text { - Je réponds à la correspondance d'un client dans la } \\
\text { langue dans laquelle le texte (p. ex., lettre, courriel) a été } \\
\text { rédigé. } \\
\text { - Dans ma correspondance, il y a des moyens pour } \\
\text { faire connaître que j'offre des services en français } \\
\text { et en anglais (p. ex., papier en-tête, signature, titre } \\
\text { professionnel } \\
\text { - Je rédige mes correspondances en anglais à moins que } \\
\text { le client ne le demande autrement. } \\
\text { - Lorsque je prépare des ressources informationnelles et } \\
\text { éducationnelles à l'intention des clients, je les prépare en } \\
\text { français ou en français et en anglais (p. ex., documents } \\
\text { écrits, présentations) }\end{array}$ & $\begin{array}{l}\text { Énoncé éliminé, stabilité temporelle problématique } \\
\text { Énoncé négatif - éliminé, stabilité temporelle problématique } \\
\text { Énoncé déplacé avec «Interventions spécialisées », stabilité } \\
\text { temporelle et taux de réponse «N/A » problématiques }\end{array}$ \\
\hline $\begin{array}{l}\text { Intervention } \\
\text { Dans mon intervention en français auprès d'un client } \\
\text { francophone... } \\
\text { • je reprends certaines expressions et un vocabulaire } \\
\text { utilisés par le client pour qu'il se sente à l'aise de parler } \\
\text { en français avec moi. }\end{array}$ & $\begin{array}{l}\text { Énoncé déplacé avec « accueil et prise en charge », } \\
\text { problématique pour la cohérence interne de la sous-échelle } \\
\text { initiale }\end{array}$ \\
\hline $\begin{array}{l}\text { - } 4 \text { énoncés présentaient un fort taux de réponse « ne } \\
\text { s'applique pas ». }\end{array}$ & $\begin{array}{l}\text { Interventions spécialisées } \\
\text { Cette section a été ajoutée pour évaluer certains comportements } \\
\text { qui ne font pas partie des tâches de tous les intervenants. Elle } \\
\text { permet un choix de réponse «ne s'applique pas », mais ces } \\
\text { énoncés ne sont pas inclus dans le calcul du score total de la } \\
\text { section « intervention» }\end{array}$ \\
\hline $\begin{array}{l}\text { Soutien et aiguillage } \\
\text { II m'arrive de diriger un client francophone vers un } \\
\text { autre intervenant (demande de consultation, transfert } \\
\text { d'établissement, etc.)? Si oui : } \\
\text { - je le dirige vers un intervenant disponible, peu importe sa } \\
\text { langue d'usage. }\end{array}$ & Énoncé négatif—éliminé \\
\hline
\end{tabular}




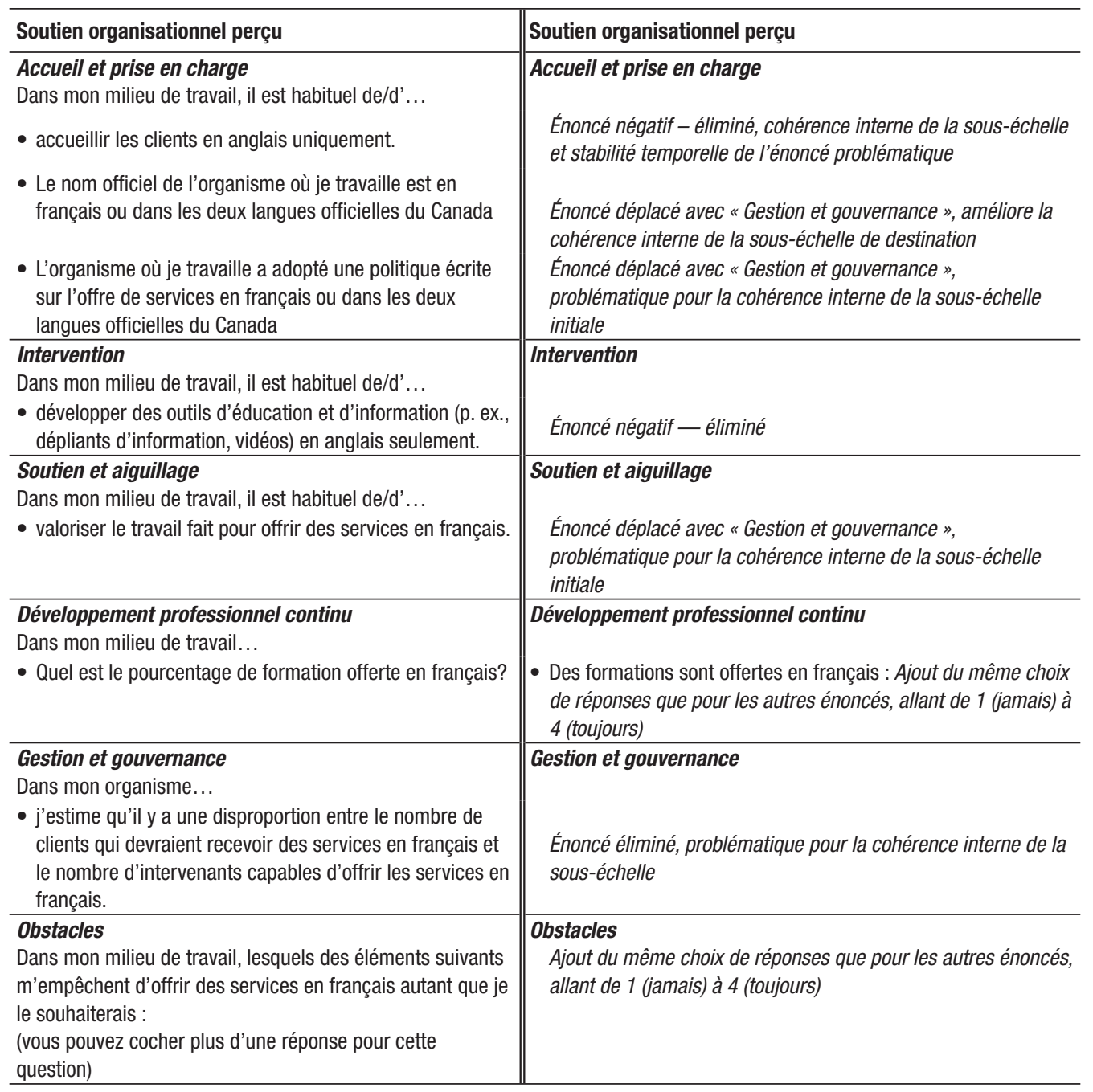

Le Tableau 4 présente les changements apportés à la suite de l'évaluation de la version expérimentale de l'outil. Parmi les quatre énoncés formulés négativement, deux obtenaient une faible stabilité temporelle. Puisque nous n'avons pas observé de phénomène de réponses automatiques (aucun répondante ou 
répondant n'ayant choisi la même réponse à tous les énoncés ou des réponses contradictoires à un énoncé formulé négativement et à son contraire formulé positivement), tous les énoncés formulés à la négative ont été retirés de l'échelle. Deux autres énoncés problématiques pour la stabilité temporelle ont été retirés, ce qui ne nuisait pas à la cohérence interne de la sous-échelle et permettait d'en améliorer la stabilité temporelle.

De même, un énoncé problématique pour la cohérence interne de sa sous-échelle initiale a été éliminé, car le déplacer n'améliorait pas les qualités de l'outil. Cinq énoncés ont été déplacés dans une sous-échelle différente de celle où ils avaient été placés initialement. Ce changement faisait augmenter la consistance interne de sous-échelle initiale ou améliorait celle de leur souséchelle de destination, ou les deux.

Puisque plusieurs énoncés qui étaient jugés comme n'étant pas applicables lors de la première passation obtenaient des réponses à la seconde passation, forcer une réponse pourrait probablement augmenter la stabilité temporelle du questionnaire. En effet, on observe que les scores pondérés ont en général une meilleure stabilité temporelle que les scores bruts (vrai pour 7 sous-échelles sur 10). Ainsi, il a été décidé de retirer le choix de réponse "ne s'applique pas " et de créer des échelles facultatives pour les interventions spécialisées et l'aiguillage, car ces activités ne font pas partie des tâches de toutes les intervenantes et de tous les intervenants en santé ou en service social. Cinq énoncés ont été déplacés dans cette nouvelle sous-échelle intitulée « interventions spécialisées ", sous-échelle facultative à l'image de celle sur le soutien et l'aiguillage qui n'était remplie que par les personnes qui faisaient ce type d'intervention.

Enfin, certains énoncés ont été reformulés (changements indiqués en caractères italiques dans l'Annexe), les questions ouvertes ont été éliminées et les questions sur l'offre de formation en français et sur les obstacles ont été reformulées pour qu'on y réponde sur la même échelle que l'ensemble des autres énoncés, ce qui facilitera l'analyse des résultats dans le futur. 


\section{Évaluation de la Mesure de l'offre active de services en français en contexte minoritaire, version 1.0}

Les caractéristiques métrologiques de chacune des nouvelles sous-échelles ont été calculées avec les énoncés tels qu'ils ont été répondus par nos participantes et participants, c'est-à-dire, sans les reformulations et les modifications aux choix de réponses proposés. Les résultats sont présentés au Tableau 5.

Tableau 5 - Caractéristiques de la

Mesure de l'offre active de services en français en contexte minoritaire, version 1.0

\begin{tabular}{|c|c|c|c|c|}
\hline Échelle et sous-échelle & $\begin{array}{c}\text { Nombre } \\
\text { d'énoncés }\end{array}$ & $\begin{array}{l}\text { Score total } \\
\text { Moy (é.t. }\end{array}$ & CCI de l'échelle & $\alpha$ de l'échelle \\
\hline \multicolumn{5}{|c|}{ Échelle des comportements d'offre active } \\
\hline $\begin{array}{l}\text { Accueil/prise en charge } \\
\text { Score brut }{ }^{1} \\
\text { Score pondéré }{ }^{1}\end{array}$ & 10 & $\begin{array}{c}(\mathrm{n}=60) \\
23,1 / 40(8,5(57,8 \%) 28,5 / 40 \\
(8,0(71,1 \%) \\
\end{array}$ & $\begin{array}{l}(\mathrm{n}=22) \\
0,644 \\
0,589\end{array}$ & $\begin{array}{c}(\mathrm{n}=56) \\
0,743\end{array}$ \\
\hline $\begin{array}{l}\text { Intervention } \\
\text { Score brut } \\
\text { Score pondéré }\end{array}$ & 4 & $\begin{array}{c}(\mathrm{n}=55 / 53) \\
11,4 / 16(4,4(71,3 \%) \\
13,1 / 16(3,0(81,9 \%) \\
\end{array}$ & $\begin{array}{c}(\mathrm{n}=22) \\
0,587 \\
0,843\end{array}$ & $\begin{array}{r}(\mathrm{n}=53) \\
0,752\end{array}$ \\
\hline $\begin{array}{l}\text { Intervention spécialisée } \\
\text { Score brut } \\
\text { Score pondéré }{ }^{2} \\
\end{array}$ & 5 & $\begin{array}{c}(\mathrm{n}=58 / 53) \\
8,6 / 20(5,0(40,0 \%) \\
14,0 / 20(4,6(70,0 \%) \\
\end{array}$ & $\begin{array}{c}(n=20) \\
0,476 \\
0,602 \\
\end{array}$ & $\begin{array}{c}(\mathrm{n}=52) \\
0,597\end{array}$ \\
\hline $\begin{array}{l}\text { Soutien et aiguillage } \\
\text { Score brut } \\
\text { Score pondéré }\end{array}$ & 4 & $\begin{array}{c}(\mathrm{n}=32) \\
12,1 / 16(3,6(75,6 \%) \\
12,8 / 16(3,4(80,0 \%) \\
\end{array}$ & $\begin{array}{c}(\mathrm{n}=12) \\
0,701 \\
0,916\end{array}$ & $\begin{array}{c}(\mathrm{n}=32) \\
0,743\end{array}$ \\
\hline $\begin{array}{l}\text { Total : comportements d'offre active } \\
\text { Score brut } \\
\text { Score pondéré }{ }^{2}\end{array}$ & 23 & $\begin{array}{c}(n=60 / 55) \\
48,4 / 92(20,5(52,6 \%) \\
67,2 / 92(16,3(73,0 \%)\end{array}$ & $\begin{array}{c}(\mathrm{n}=22) \\
0,685 \\
0,744\end{array}$ & $\begin{array}{c}(\mathrm{n}=31) \\
0,861\end{array}$ \\
\hline \multicolumn{5}{|l|}{ Échelle du soutien organisationnel perçu } \\
\hline $\begin{array}{l}\text { Accueil/prise en charge } \\
\text { Score brut } \\
\text { Score pondéré }\end{array}$ & 12 & $\begin{array}{c}(\mathrm{n}=52) \\
32,2 / 48(8,1(67,1 \%) \\
38,6 / 48(8,1(80,3 \%)\end{array}$ & $\begin{array}{c}(\mathrm{n}=22) \\
0,683 \\
0,849\end{array}$ & $\begin{array}{c}(\mathrm{n}=49) \\
0,816\end{array}$ \\
\hline $\begin{array}{l}\text { Intervention } \\
\text { Score brut } \\
\text { Score pondéré } \\
\end{array}$ & 6 & $\begin{array}{c}(\mathrm{n}=51) \\
12,8 / 24(6,0(53,3 \%) \\
16,0 / 24(4,8(66,7 \%) \\
\end{array}$ & $\begin{array}{c}(\mathrm{n}=22 / 21) \\
0,680 \\
0,866 \\
\end{array}$ & $\begin{array}{c}(\mathrm{n}=51) \\
0,794\end{array}$ \\
\hline $\begin{array}{l}\text { Soutien et aiguillage } \\
\text { Score brut } \\
\text { Score pondéré } \\
\end{array}$ & 3 & $\begin{array}{c}(\mathrm{n}=51) \\
6,8 / 12(3,7(56,7 \%) \\
6,9 / 12(3.7(57,3 \%) \\
\end{array}$ & $\begin{array}{c}(\mathrm{n}=22 / 20) \\
0,601 \\
0,271 \\
\end{array}$ & $\begin{array}{c}(\mathrm{n}=50) \\
0,761\end{array}$ \\
\hline $\begin{array}{l}\text { Développement professionnel continu }{ }^{3} \\
\text { Score brut } \\
\text { Score pondéré }\end{array}$ & 7 & $\begin{array}{c}\mathrm{n}=50 / 43) \\
9,9 / 28(6.2(35,4 \%) \\
14,3 / 28(6,7(51,1 \%)\end{array}$ & $\begin{array}{c}\mathrm{n}=21 / 18) \\
0,494 \\
0,638\end{array}$ & $\begin{array}{c}(\mathrm{n}=49) \\
0,851\end{array}$ \\
\hline
\end{tabular}




\begin{tabular}{|c|c|c|c|c|}
\hline Échelle et sous-échelle & $\begin{array}{c}\text { Nombre } \\
\text { d'énoncés }\end{array}$ & $\begin{array}{l}\text { Score total } \\
\text { Moy (é.t. }\end{array}$ & CCI de l'échelle & $\alpha$ de l'échelle \\
\hline $\begin{array}{l}\text { Gestion et gouvernance } \\
\text { Score brut } \\
\text { Score pondéré }\end{array}$ & 7 & $\begin{array}{c}(\mathrm{n}=52) \\
17,4 / 28(6,2(62,1 \%) 22,3 / 28 \\
(5,5(79,7 \%)\end{array}$ & $\begin{array}{l}(\mathrm{n}=22) \\
0,782 \\
0,923\end{array}$ & $\begin{array}{l}(\mathrm{n}=51) \\
0,703\end{array}$ \\
\hline $\begin{array}{l}\text { Total : soutien organisationnel } \\
\text { Score brut } \\
\text { Score pondéré }{ }^{2}\end{array}$ & 35 & $\begin{array}{c}(\mathrm{n}=52 / 50) \\
78,3 / 140(21,9(55,9 \%) \\
98,3 / 140(21,8(70,2 \%)\end{array}$ & $\begin{array}{c}(\mathrm{n}=22 / 21) \\
0,737 \\
0,867\end{array}$ & $\begin{array}{c}(n=44) \\
0,898\end{array}$ \\
\hline $\begin{array}{l}\text { Obstacles (absent ou présent) }{ }^{4} \\
\text { Score brut }\end{array}$ & 7 & $\begin{array}{c}(\mathrm{n}=51) \\
0,63 / 6(1,1\end{array}$ & $\begin{array}{l}(\mathrm{n}=22) \\
0,605\end{array}$ & $\begin{array}{r}\text { Ne peut se } \\
\text { calculer }\end{array}$ \\
\hline
\end{tabular}

Exemple du calcul, pour une personne qui a fourni 8 réponses sur 10 énoncés dans l'échelle et pour laquelle la somme des 8 réponses est de 25

- le calcul du score brut est : 25/40 (où 40 est le maximum de l'échelle et la réponse manquante se voit attribuer la cote "zéro ")

— le calcul du score pondéré est : $\underline{25} \times 40=31,25 / 40$

(où 32 est le maximum possible en n'utilisant que les énoncés notés)

2 Le score brut pour cette sous-échelle ne signifie pas absence d'offre active, mais absence des tâches incluses dans l'échelle. Le score pondéré sera un meilleur indicateur des comportements d'offre active.

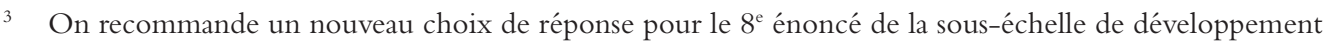
continu, mais comme nous n'avons pas de données empiriques à ce sujet, les calculs ici ont été faits avec 7 énoncés.

4 On recommande un nouveau choix de réponse pour toute la sous-échelle des obstacles, mais comme nous n'avons pas de données empiriques à ce sujet, les calculs n'ont pu être refaits.

Les changements ont permis d'améliorer les qualités métrologiques de l'outil. La consistance interne est maintenant acceptable ( $\alpha$ entre 0,70 et 0,90$)$ pour toutes les sous-échelles, à l'exception de la sous-échelle facultative sur les interventions spécialisées. La stabilité temporelle est améliorée pour les deux sous-échelles qui étaient au départ les plus problématiques. Il n'y a plus d'indication de biais systématiques. Les résultats obtenus demeurent inférieurs aux valeurs souhaitées (CCI >0,60) pour certains scores bruts ou pondérés, mais s'en rapprochent à l'exception du score pondéré de la sous-échelle "Soutien organisationnel : soutien et aiguillage ». Il est possible que les modifications proposées à certaines formulations et aux choix de réponses améliorent la stabilité des échelles, mais pour le vérifier il faudrait une nouvelle étude où le nouveau questionnaire serait soumis à un groupe de répondantes et de répondants. 
Les analyses de corrélation soutiennent la validité de construit du questionnaire. Les corrélations entre le score de chaque sous-échelle et le total de son échelle sont toutes supérieures à 0,70 , à l'exception de la sous-échelle de soutien et aiguillage du soutien organisationnel qui est plus faiblement corrélée au total du soutien organisationnel $(0,32$ pour le score pondéré - résultats non montrés). Des corrélations modérées sont observées entre les résultats de l'échelle des comportements d'offre active des intervenantes et des intervenants et celle du soutien organisationnel perçu, ce qui signifie que ces échelles mesurent des concepts apparentés, bien que différents (Tableau 6). Ce résultat est conforme à ce qui était attendu en théorie.

Tableau 6 - Corrélations entre les sous-échelles de comportements d'offre active et les sous-échelles correspondantes du soutien organisationnel

\begin{tabular}{|l|l|c|c|c|}
\hline \multicolumn{1}{|c|}{$\begin{array}{c}\text { Comportements d'offre } \\
\text { active }\end{array}$} & $\begin{array}{c}\text { Soutien organisationnel } \\
\text { perçu }\end{array}$ & $\mathbf{n}$ & $\begin{array}{c}\text { Corrélation de } \\
\text { Pearson }\end{array}$ & Signification (p) \\
\hline Accueil et prise en charge & Accueil et prise en charge & 52 & 0,580 & 0,000 \\
\hline Intervention & Intervention & 48 & 0,416 & 0,003 \\
\hline Soutien et aiguillage & Soutien et aiguillage & 31 & 0,547 & 0,001 \\
\hline Total & Total & $\mathbf{4 9}$ & $\mathbf{0 , 6 0 5}$ & $\mathbf{0 , 0 0 0}$ \\
\hline
\end{tabular}

\section{Discussion}

Le but de la présente étude était d'effectuer une première évaluation empirique de la Mesure de l'offre active de services en français en contexte minoritaire et de bonifier l'outil en fonction des résultats obtenus. Ces derniers ont permis de créer une mesure qui présente certaines forces dont une bonne consistance interne pour chacune des sous-échelles, une indication de sa validité de construit par des corrélations modérées entre les comportements individuels et les indicateurs de soutien organisationnel en lien avec ces mêmes comportements, ainsi qu'une bonne variabilité des réponses et l'absence d'effet plafond. En effet, le résultat moyen 
"S'il est souhaité de disposer d'un outil permettant de mesurer l'évolution des comportements d'offre active à la suite d'activités de formation ou de changements organisationnels en faveur de services en français, d'autres études sont nécessaires avant de pouvoir utiliser à cette fin la Mesure de l'offre active de services en français en contexte minoritaire. " n'atteint jamais le maximum possible pour l'échelle malgré un échantillon très francophone qui travaille majoritairement dans des milieux s'engageant à offrir des services en français.

Les analyses utilisant le score pondéré suggèrent que la stabilité temporelle de l'outil est satisfaisante pour 8 des 10 sous-échelles, mais demeure mitigée pour deux d'entre elles. Il faudra être prudent dans l'interprétation de ces résultats. En raison de la faible proportion de répondantes ou de répondants ayant répondu au questionnaire à deux reprises, ainsi qu'en raison des modifications apportées à l'outil (élimination du choix «ne s'applique pas »), il sera utile de poursuivre les études de sa stabilité temporelle afin d'augmenter la confiance envers les résultats obtenus. La poursuite de l'étude de cette propriété auprès d'un plus grand échantillon, soit une cinquantaine de participantes ou participants, permettrait de déterminer les coefficients de fidélité avec une plus grande précision ainsi que le changement minimal qui peut être considéré comme un changement réel plutôt que comme une erreur de mesure (Lexell et Downham, 2005; Streiner et Norman, 2008). Il faut aussi noter que les coefficients de corrélation intraclasse sont sensibles à la variance de l'échantillon et que les coefficients obtenus peuvent être artificiellement bas lorsque les variances entre la première et la seconde passation ne sont pas similaires (Schuck, 2004), ce qui pourrait avoir une légère influence dans le cas de la présente étude.

Cette étude démontre que le développement d'un tel outil présente certains défis et confirme la nécessité de procéder en plusieurs étapes, comme mentionné dans les écrits méthodologiques (Bradburn 2004; Streiner et Norman, 2008). S'il est souhaité de disposer d'un outil permettant de mesurer l'évolution des comportements d'offre active à la suite d'activités de formation ou de changements organisationnels en faveur de services en français, d'autres études sont nécessaires avant de pouvoir utiliser à cette fin la Mesure de l'offre active de services en français en contexte minoritaire. Dans l'état actuel de son développement, cette dernière peut être utilisée comme un outil d'autoévaluation pour les personnes qui souhaiteraient se situer par rapport à un ensemble possible de comportements d'offre active. Cette autoévaluation 
peut entrainer une première sensibilisation aux mécanismes de l'offre active qui peut conduire à l'amélioration de ses propres comportements ou à des interventions auprès de son organisation visant l'implantation de pratiques qui la facilitent.

Il sera aussi utile de disposer d'une mesure valide de l'offre active de services en français afin d'en étudier les déterminants. En effet, les résultats de notre recension d'écrits nous amènent à croire que des éléments de soutien organisationnel (Bouchard, et collab. 2009;2010), de vitalité ethnolinguistique, d'identité, de motivation langagière (Landry, et collab., 2006; 2008), de compétences linguistiques et culturelles (Betancourt, et collab., 2003) pourraient influencer la propension d'une personne à s'afficher comme parlant français et à offrir activement des services en français. Ces éléments constituent des déterminants probables de l'offre active de services en français. Pouvoir mesurer quantitativement et simultanément la présence de comportements d'offre active et la présence de ces déterminants probables permettrait une meilleure connaissance de l'impact de ces derniers sur le comportement des professionnelles et professionnels de la santé et des services sociaux. Cela devrait contribuer à mieux cibler les éléments sur lesquels il est possible d'agir pour mieux les outiller et cette connaissance pourrait alimenter les efforts de création de nouveaux contenus de formation. C'est ce que visait l'étude plus large mentionnée au début du présent article. Cette dernière n'a toutefois pas donné tous les résultats escomptés puisque l'échantillon était trop homogène, entre autres, sur le plan du profil psycholinguistique. Il a donc été impossible de vérifier l'impact de différents profils psycholinguistiques sur la propension vers l'offre active. Par ailleurs, certaines différences ont été observées dans les caractéristiques des milieux de travail des répondantes et des répondants. Ainsi, dans une analyse secondaire des données recueillies par notre équipe, Brulé, et collab. (2013) ont pu étudier les liens entre des caractéristiques suivantes et les comportements d'offre active de la part des intervenantes et des intervenants : province de travail, langue du milieu de travail, stratégie la plus utilisée pour servir la clientèle francophone, rôle principal (clinique ou administratif) et score total pondéré du soutien organisationnel à l'offre active. 
Lorsque l'influence de ces variables a été analysée simultanément dans une analyse de régression linéaire, seul le total pondéré du soutien organisationnel est ressorti comme déterminant des comportements d'offre active des intervenantes et intervenants. Ce facteur expliquait $35 \%$ de la variance observée dans les comportements individuels. Ces résultats sont à mettre en lien avec ceux qu'avaient obtenus Bouchard et Vézina (2009), de manière qualitative, auprès d'intervenantes et intervenants francophones œuvrant en milieu anglo-dominant. Les attitudes ambigües ou négatives de l'employeur ou des collègues représentaient des défis importants pour mettre en pratique l'offre de services en français par ces francophones. Cela met en évidence le rôle de leadership que sont appelés à jouer les gestionnaires afin de mieux répondre aux besoins des francophones vivant en situation minoritaire. Par des formations, des activités de sensibilisation ou par leurs attitudes, ils peuvent favoriser le développement de compétences linguistiques et culturelles et contribuer à transformer considérablement la culture organisationnelle. Le soutien organisationnel perçu est d'ailleurs reconnu comme un déterminant important de l'engagement affectif d'un employé envers l'organisation pour laquelle il travaille (Vandenberghe, 2005) et est donc essentiel à toute organisation qui voudrait améliorer son offre de services en français en contexte minoritaire.

\section{Limites de l'étude}

La plus grande limite de l'étude est certainement son faible taux de réponse. Si le nombre de répondantes et de répondants est suffisant pour l'étude de la distribution des réponses et de la consistance interne, il est plus faible pour l'étude de la stabilité temporelle de l'outil. De plus, cette faible participation des personnes sollicitées pour répondre au questionnaire affecte la représentativité des données. Bien que nous ne disposions pas de données précises sur les caractéristiques de l'ensemble des personnes à qui le questionnaire a été envoyé, il est permis de croire que ce groupe de diplômées ou de diplômés comporte des gens d'origine un peu plus variée que celle des répondantes ou répondants. La relative homogénéité du groupe sur le plan linguistique limite la possibilité 
«...cette étude constitue à notre connaissance une première tentative d'évaluation des comportements d'offre active de services en français de la part d'intervenantes et d'intervenants de la santé et des services sociaux. Bien que réalisée auprès d'un échantillon partiellement représentatif, l'étude a permis de déceler certains des problèmes de la version expérimentale et de proposer une version améliorée. " de généraliser les résultats à d'autres groupes. Il est intéressant de constater que $40 \%$ des participantes et participants (50\% de ceux provenant du Nouveau-Brunswick et $38 \%$ de ceux de l'Ontario) ont indiqué offrir des services en français à plus de $60 \%$ de leur clientèle. En Ontario du moins, aucune communauté n'a une proportion de $60 \%$ de francophones. L'étude a donc rejoint majoritairement des personnes qui travaillent déjà beaucoup à servir leurs concitoyens francophones. Il n'est pas certain qu'un échantillon constitué de personnes francophiles moins exposées au français ou de francophones travaillant dans des milieux plus typiquement anglophones réagiraient de la même façon devant les énoncés qui leur sont proposés, ou même qu'ils les comprendraient de la même façon.

De plus, l'échantillon comportait 8,3\% de participantes ou participants provenant de milieux qui offrent exclusivement des services en français. Bien que dans ces milieux l'intervenante ou l'intervenant n'a pas à faire l'effort d'offrir des services en français puisqu'ils sont déjà offerts dans cette langue, ces services doivent tout de même être adaptés à une clientèle en contexte minoritaire. Pour ces répondantes ou répondants, certaines questions de la section " accueil et prise en charge » étaient peut-être moins pertinentes. Par contre, la majorité des questions demeurent justifiées, comme celles liées à l'adaptation du niveau de langue ou à l'aiguillage des clientes ou clients vers des services en français. La participation de ces personnes a pu augmenter la variabilité des contextes représentés, ce qui est un atout pour l'évaluation des qualités métrologiques de l'outil.

Enfin, les consignes de certaines questions sur les caractéristiques sociodémographiques des participantes et des participants pouvaient être ambigües. Par exemple, bien que nous ayons demandé aux 13,3 \% de personnes qui travaillent au Québec de se référer à une expérience dans une autre province, nous n'avions aucun moyen de le vérifier et devions nous fier à leur bonne compréhension. De même, la question qui demandait dans quelle(s) langue(s) officielle(s) leur milieu de travail s'engage à offrir des services peut avoir été perçue de différentes façons. Ces limites réduisent la précision de la description de l'échantillon, 
mais ne devraient pas modifier de façon substantielle les résultats relatifs aux qualités métrologiques de l'outil.

Malgré ces limites, cette étude constitue à notre connaissance une première tentative d'évaluation des comportements d'offre active de services en français de la part d'intervenantes et d'intervenants de la santé et des services sociaux. Bien que réalisée auprès d'un échantillon partiellement représentatif, l'étude a permis de déceler certains des problèmes de la version expérimentale et de proposer une version améliorée.

\section{Conclusion}

Cette démarche a permis d'examiner la fidélité, en particulier la consistance interne et la stabilité temporelle, d'un outil susceptible d'aider les professionnelles et professionnels, les éducateurs et éducatrices, les gestionnaires ainsi les chercheuses et chercheurs à mesurer les comportements d'offre active. Elle a aussi permis de corriger les erreurs de conception de l'outil qui nuisaient au calcul d'un score total et de proposer une version améliorée de la Mesure de l'offre active de services en français en contexte minoritaire. Si l'outil peut encore être amélioré, il s'avère que cette version fournit des informations clés pour la poursuite de la recherche sur la mesure et l'évolution de ces comportements.

Pour l'avenir, l'étude de la stabilité temporelle de la nouvelle version de l'outil présenté dans cet article est certainement à l'ordre du jour. De plus, notre équipe mène actuellement une nouvelle recherche pour tenter d'identifier les liens possibles entre les profils psycholinguistiques et les comportements d'offre active, cette fois-ci auprès de résidents en médecine. En recrutant les participantes et participants auprès de trois universités ayant des contextes linguistiques diversifiés, nous espérons obtenir la variabilité nécessaire à la conduite des analyses des déterminants de l'offre active de services en français. Enfin, la Mesure de l'offre active de services en français en contexte minoritaire a été conçue sous la forme d'une autoévaluation. Dans des recherches subséquentes, il serait aussi intéressant de créer des grilles d'observation des 
comportements d'offre active qui pourraient être utilisées par les précepteurs et préceptrices de stage ou encore en salle de classe lors d'activités de jeux de rôle ou scénarios simulés. Les énoncés contenus dans la Mesure de l'offre active de services en français en contexte minoritaire pourraient servir de point de départ pour ces recherches puisqu'elle fournit de bons exemples de comportements d'offre active. Si de telles grilles d'observations sont créées, elles devront subir une évaluation de leurs propriétés métrologiques dans un contexte de formation. De telles études sont nécessaires dans le contexte des efforts d'amélioration des pratiques de l'offre active de services en français au sein des communautés francophones en situation minoritaire. Il existe encore plusieurs lacunes dans la prestation et l'organisation de ces services auxquelles il faudra remédier au cours des prochaines années.

\section{Bibliographie}

BENOÎT,Josée, et collab. (2014 [accepté]). «Les approches pédagogiques et les contenus de formation dans les programmes de santé et de service social en milieu minoritaire francophone au Canada ", Minorités linguistiques et sociétés.

BETANCOURT, Joseph R., et collab. (2003). «Defining cultural competence: a practical framework for addressing racial/ethnic disparities in health and health care ", Public Health Reports, Vol. 118, No 4, p. 293-302.

BOUCHARD, Louise, Marielle BEAULIEU et Martin DESMEULES (2012). "L'offre active de services de santé en français en Ontario : une mesure d'équité ", Reflets, Revue d'intervention sociale et communautaire, Vol. 18, № 2, p. 38-65. DOI: 10.7202/1013173ar

BOUCHARD, Louise, et collab. (2009). "La santé en situation linguistique minoritaire ", Healthcare Policy, Vol. 4, No 4, p. 33-40.

BOUCHARD, Pier, Sylvain VÉZINA et Mylène SAVOIE (2010). Rapport du Dialogue sur l'engagement des étudiants et des futurs professionnels pour de meilleurs services de santé en français dans un contexte minoritaire : Formation et outillage, Recrutement et rétention, Ottawa, Consortium national de formation en santé, $37 \mathrm{p}$.

BOUCHARD, Pier, et SylvainVÉZINA (2009). L'outillage des étudiants et des nouveaux professionnels : une condition essentielle à l'amélioration des services de santé en français, Ottawa, Consortium national de formation en santé, $66 \mathrm{p}$.

BRADBURN, Normand, Seymour SUDMAN et Brian WANSINK (2004). Asking questions: the definitive guide to questionnaire design, San Francisco, Jossey-Bass, 448 p.

BRÛLÉ, Stéphanie, et collab. (2013). Examen du lien entre les caractéristiques du milieu de travail et les caractéristiques sociodémographiques des intervenants de la santé et des services sociaux, et leurs comportements d'offre active en français, Essai rédigé sous la direction de Lynn Casimiro, dans le cadre 
de l'obtention du grade de maitrise en Physiothérapie, École des Sciences de la Réadaptation, Université d'Ottawa.

CARDINAL, Linda, et Anik SAUVÉ (2010). De la théorie à la pratique :les mécanismes d'offre des services en français dans le domaine de la justice en Ontario, Volume 1, Ottawa, Chaire de recherche sur la francophonie et les politiques publiques, $43 \mathrm{p}$.

CHARLAND, Francine, et collab. (2011). Cadre référentiel de compétences linguistiques et culturelles à l'intention des organismes du Nord-Est de l'Ontario, élaboré pour le Réseau régional de langue française de Meilleur départ du Nord-Est de l'Ontario grâce au financement de Patrimoine Canada, réf. du 18 mai 2012, http://www.entitesante2.ca/wp-content/uploads/2012/05/Cadre-R\%C3\%A9f\%C3\%A9 rentiel1.pdf

COMITÉ SANTÉ EN FRANÇAIS DE FREDERICTON (2006). Évaluation de l'offre active des services de santé de première ligne, Fredericton, Groupe Progecom communication \& stratégies d'entreprise, $15 \mathrm{p}$.

CONSORTIUM NATIONAL DE FORMATION EN SANTÉ (CNFS) (2011). À propos de nous : Mission, valeurs et mandat, réf. du 17 décembre 2012, http://cnfs.net/fr/mission.php

CONSORTIUM NATIONAL DE FORMATION EN SANTÉ (CNFS) (2012). Cadre de référence pour la formation à l'offre active des services de santé en français, Ottawa, Consortium national de formation en santé, $27 \mathrm{p}$.

CONSORTIUM POUR LA PROMOTION DES COMMUNAUTÉS EN SANTÉ (2011). Collaborer avec les francophones en Ontario : de la compréhension du contexte à l'application des pratiques prometteuses, Ontario, Nexus Santé.

DROLET, Marie, et collab. (2014). "Health Services for Linguistic Minorities in a Bilingual Setting: Challenges for Bilingual Professionals ", Qualitative Health Research, Vol. 24, No 3, p. 295-305.

DROUIN, Jeanne, et Christine RIVET (2003). «Training medical students to communicate with a linguistic minority group ", Academic Medicine, Vol. 78, № 6, p. 599-604.

DUBOULOZ, Claire-Jehanne, et collab. (2014 [accepté]). «Proposition de lignes directrices pour la formation des futurs professionnels en santé et en service social œuvrant en situation francophone minoritaire ", Reflets : revue d'intervention sociale et communautaire, Vol. 20, $\mathrm{N}^{\circ} 2$.

EVANS, Robert G., Morris L. BARER et Theodor R. MATMOR (dirs.) (1996). Etre ou ne pas être en bonne santé. Biologie et déterminants sociaux de la maladie, Paris, John Libbey Eurotext, et Montréal, Les Presses de l'Université de Montréal, 359 p.

FOR GUES, Éric, et collab. (2011). L'offre de services de santé en français en contexte francophone minoritaire, Moncton, Nouveau-Brunswick, Institut canadien de recherche sur les minorités linguistiques, $182 \mathrm{p}$.

GARDNER, Paul L. (1995). "Measuring attitudes to sciences: Unidimensionality and internal consistency revisited ", Research in Sciences Education,Vol. 25, No 3, 283-289.

GOUVERNEMENT DE L'ÎLE-DU-PRINCE-ÉDOUARD (2011). L'offre active de services en français et anglais, réf. du 13mai 2011, www.bonjour-hello.ca/fr/?cat=102\&catProv=PE

GOUVERNEMENT DU MANITOBA (2011). Directive no. 1 - concept de l'offre active, réf. du 13 mai 2011,www.bonjour-hello.ca/DATA/MB/MEDIA/ Part_II_Guidelines_1_Active_Offer_ Concept.pdf 
LANDIS, J. Richard, et Gary G. KOCH (1977). "The measurement of observer agreement for categorical data ", Biometrics, Vol. 33, No 1, p. 159-174.

LANDRY, Rodrigue, Kenneth DEVEAU et Réal ALLARD (2006). «Vitalité ethnolinguistique et construction identitaire : le cas de l'identité bilingue ", Éducation et francophonie,Vol. 34, p. 54-81.

LANDRY, Rodrigue, Réal ALLARD et Kenneth DEVEAU (2008). "Un modèle macroscopique du développement psycholangagier en contexte intergroupe minoritaire ", Diversité urbaine, numéro hors série, p. 45-68.

LEXELL, Jan E., et David Y. DOWNHAM (septembre 2005). "How to assess the reliability of measurements in rehabilitation ", American Journal of Physical Medicine and Rehabilitation,Vol. 84, No 9, p. 719-723.

NUNNALLY, Jum C., et Ira H. BERNSTEIN (1994). Psychometric Theory, 3e edition, New York, McGraw-Hill, 736 p.

ORGANISATION MONDIALE DE LA SANTÉ - COMMISSION DES DÉTERMINANTS SOCIAUX DE LA SANTÉ (2008). Combler le fossé en une génération: Instaurer l'équité en santé en agissant sur les déterminants sociaux de la santé, Genève, Organisation mondiale de la santé (OMS), réf. du 17 décembre 2012, http://www.whqlibdoc.who.int/publications/2009/9789242563702_fre. pdf

RÉSEAU DES SERVICES DE SANTÉ EN FRANÇAIS DE L'EST DE L'ONTARIO (2010). La désignation des établissements de santé dans l'Est ontarien-Mise en contexte, Ottawa, [inédit].

SANTÉ CANADA - COMITÉ CONSULTATIF DES COMMUNAUTÉS FRANCOPHONES EN SITUATION MINORITAIRE (2007). Pour un nouveau leadership en matière d'amélioration des services de santé en français, [rapport au ministre fédéral de la santé], Ottawa, Santé Canada, 58 p.

SAVARD, Jacinthe, et collab. (2014 [accepté]). «Conception d'une mesure de l'offre active de services sociaux et de santé en français en contexte minoritaire ", Minorités linguistiques et sociétés.

SCHUCK, Peter (2004). «Assessing reproducibility for interval data in health-related quality of life questionnaires: Which coefficient should be used? ", Quality of Life Research, Vol. 13, p. 571-586.

SOCIÉTÉ SANTÉ EN FRANÇAIS (2012). Destination 2018 : qualité, sécurité et mieux-être en français, Ottawa, Société santé en français, $18 \mathrm{p}$.

SOCIÉTÉ SANTÉ EN FRANÇAIS - RÉSEAU D’EXPERTISES (2010). Modèles de promotion de l'offre active et de la demande de services en français, Ottawa, Société Santé en français.

SOCIÉTÉ SANTÉ EN FRANÇAIS (2007). Santé en français, communautés en santé : Une offre active de services en santé pour une meilleure santé des francophones en situation minoritaire - Résumé des Plans directeurs 2008-2013, Ottawa, Société santé en français, réf. du 13 mai 2011, www. reseausantealbertain.ca/fr/publications/SSF_Plans_directeurs_Sommaire_2007-11-26.pdf

SOCIÉTÉ SANTÉ ET MIEUX-ÊTRE AU NOUVEAU-BRUNSWICK - Réseau-action formation et recherche (s. d.). L'offre de services dans les deux langues officielles dans le domaine de la santé, À nous d'y voir, réf. du 13 mai 2011, http://www.ssmefnb.ca/images/docs/Guide\%20 offre $\%$ 20active $\%$ 20en\%20francais.pdf

SYLVERSTEIN, B.S., W. P. FISHER et K. M. KILGORE (1992). «Applying psychometric criteria to functional assessment in medical rehabilitation: II. Defining interval measures ", Archives of Physical Medicine and Rehabilitation, Vol. 73, 507-518.

STREINER, David L., et Geoffrey R. NORMAN (2008). Health measurement scales: a practical guide to their development and use, $4^{\mathrm{e}}$ edition, Oxford, Oxford University Press, $428 \mathrm{p}$. 
TREMBLAY, Louis E., et collab. (dirs.) (2004). Répertoire des outils d'évaluation en français pour la réadaptation, Ottawa, Centre franco-ontarien de ressources pédagogiques, $585 \mathrm{p}$.

VANDENBERGHE, Christian (mai 2005). «L'engagement organisationnel dans le secteur public : quelques déterminants essentiels ", Téléscope, Vol. 12, № 4, p. 1-10.

VÉZINA, Sylvain, et collab. (2014). L'offre active de services de santé dans les deux langues officielles au Nouveau-Brunswick, Moncton, Présentation en vidéoconférence à partir du CHU Dr-GeorgesL.-Dumont.

Annexe 1 - Mesure de l'offre active de services en français en contexte minoritaire, Version $1.0^{\star 1}$

Répondez le plus honnêtement possible. Il est normal que tous les comportements énumérés dans ce questionnaire ne soient pas toujours réalisés. Répondez au questionnaire de manière à refléter votre pratique réelle.

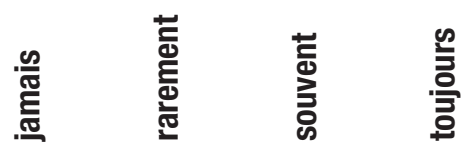

\section{Comportements d'offre active}

Accueil et prise en charge

Quelles sont les actions que je prends personnellement pour faire connaître le fait que je peux offrir des services en français?

1. Dans mon milieu de travail, je porte une identification quelconque qui indique que je peux offrir des services en français (p. ex., épinglette).

O $\quad$ O

○ $\quad 0$

O en anglais, il souhaite que je communique avec lui.

3. Lorsque j'accueille un client dont je ne connais pas la langue, je le salue en français en premier, suivi d'une salutation en anglais.

4. Dans mon milieu de travail, il y a des moyens visuels démontrant que j'offre des services en français ou dans les deux langues officielles. (p. ex., affiche sur ma porte, sur mon bureau, sur mon agenda).

5. Lorsqu'il ne le fait pas, je sensibilise mon employeur à l'importance de la promotion des services en français qui sont disponibles dans mon établissement (p. ex., affichage, publicité, site Web)

6. Lorsque je réponds au téléphone, je réponds en français en premier, suivi de l'anglais, lorsque c'est nécessaire.

$\bigcirc \quad \bigcirc \quad \bigcirc$


Répondez le plus honnêtement possible.

Il est normal que tous les comportements énumérés dans ce questionnaire ne soient pas toujours réalisés. Répondez au questionnaire de manière à refléter votre pratique réelle.

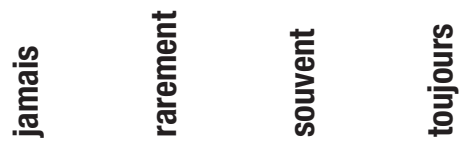

7. Le message de ma boite vocale commence par une salutation en français, suivi de l'anglais, lorsque c'est nécessaire.

$\bigcirc \quad \bigcirc \quad \bigcirc$

8. La signature de mon courriel apparaît en français en premier.

9. Je veille à ce qu'il y ait des documents informatifs et des outils de divertissement en français ( $p$. ex., dépliants, revues, journaux, radio, télévision, jeux) dans les aires communes (salle d'attente ou autre).

10. Je reprends certaines expressions et un vocabulaire utilisés par le client pour qu'il se sente à l'aise de parler en français avec moi

\section{Intervention}

Dans mon intervention en français auprès d'un client francophone...

1. je fais des démarches pour obtenir des outils d'information ou d'éducation pour les clients en français ou dans les deux langues officielles lorsqu'ils ne sont pas disponibles chez mon employeur.

2. j'utilise des outils d'éducation ou d'information (p. ex., dépliants d'information, programmes d'exercices) dont le français est adapté à ma clientèle (p. ex., contexte culturel, ethnique ou réglementaire).

3. pour l'entrevue initiale des clients francophones, j'utilise des guides ou des questionnaires dans un français adapté à ma clientèle.

4. j'aide le client à bien comprendre les énoncés lorsque les outils utilisent un français difficile à comprendre

\section{Intervention spécialisée}

1. Iorsque j'utilise des instruments de mesure standardisés (p. ex., questionnaires, échelles, inventaires), je m'assure d'utiliser des instruments validés en français (c.-à-d. dont la fidélité et la validité de la version française ont été mesurées).

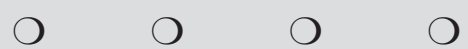

(je n'utilise pas d'instruments de mesure standardisés) 
Répondez le plus honnêtement possible.

II est normal que tous les comportements énumérés dans ce questionnaire ne soient pas toujours réalisés. Répondez au questionnaire de manière à refléter votre pratique réelle.

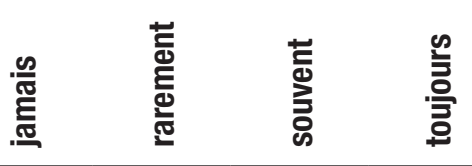

2. Iorsque j'utilise des instruments de mesure standardisés (p. ex., questionnaires, échelles, inventaires), je vérifie si les études d'évaluation comprenaient des francophones en situation minoritaire afin de mieux interpréter les résultats.

(je n'utilise pas d'instruments de mesure standardisés)

3. dans les circonstances où une activité de groupe est offerte uniquement en anglais, je trouve un moyen d'offrir une activité équivalente en français.

4. Lorsque je prépare des ressources informationnelles et éducationnelles à l'intention des clients, je les prépare en français ou en français et en anglais (p. ex., documents écrits, présentations)

5. Iorsque je supervise des stages, je demande à recevoir des stagiaires capables de parler en français afin de mieux servir ma clientèle.
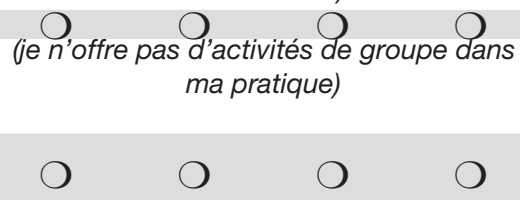

O

(je ne prépare pas de telles ressources)

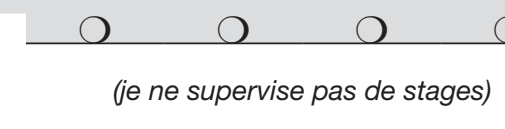

\section{Soutien et aiguillage}

II m'arrive de diriger un client francophone vers un autre intervenant (demande de consultation, transfert d'établissement, etc.)? Si oui, remplissez cette section. Sinon, passez à la prochaine section.

1. je consulte une liste à jour des employés et des organismes qui peuvent offrir des services en français.

2. j'offre au client la possibilité d'être dirigé vers un autre intervenant ou organisme francophone.

3. je précise la langue d'usage du client dans le dossier de transfert.

4. j'informe verbalement l'intervenant du choix langagier du client, ou je demande à quelqu'un de le faire.

$\begin{array}{cccc}0 & 0 & 0 & 0 \\ 0 & 0 & 0 & 0 \\ 0 & 0 & 0 & 0 \\ 0 & 0 & 0 & 0\end{array}$

\section{Soutien organisationnel perçu}

\section{Accueil et prise en charge}

Dans mon milieu de travail, il est habituel de/d'...

1. présenter des affiches en français ou dans les deux langues officielles du Canada.

2. fournir des épinglettes ou plaquettes indiquant le service disponible dans les deux langues officielles du Canada.

\begin{tabular}{rrrr}
0 & 0 & 0 & 0 \\
0 & 0 & 0 & 0 \\
\hline
\end{tabular}


Répondez le plus honnêtement possible.

Il est normal que tous les comportements énumérés dans ce questionnaire ne soient pas toujours réalisés. Répondez au questionnaire de manière à refléter votre pratique réelle.

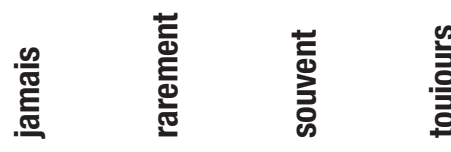

3. afficher, de façon visible et claire, la disponibilité des services en français.

4. offrir de l'information en français ou dans les deux langues officielles du Canada sur le site web de l'établissement

5. mettre à la disposition des clients des revues, des fascicules et des médias présentant l'information dans les deux langues officielles du Canada.

6. diffuser de l'information dans les journaux et à la radio en français.

7. recruter du personnel capable d'offrir des services en français à tous les paliers de services

8. tenir les réunions en français ou dans les deux langues officielles du Canada

9. faciliter l'utilisation du français entre les employés.

10. informer le client de l'engagement de l'établissement à lui offrir des services de qualité égale dans les deux langues officielles du Canada.

11. converser en français entre employés francophones.

12. imprimer le papier en-tête et les cartes d'affaires de l'organisme en français ou dans les deux langues officielles du Canada.

\section{Intervention}

Dans mon milieu de travail, il est habituel de/d'...

1. fournir des outils de travail facilitant le service en français ou dans les deux langues officielles du Canada (p. ex., calendriers, correcteurs de langue, dictionnaires, formulaires).

2. fournir un glossaire de terminologie médicale ou de services sociaux et de santé en français.

3. offrir des services d'information, de prévention ou de soins spécialisés à distance en français (p. ex., centre d'appels, Internet, vidéoconférences, télémédecine)

○ $\bigcirc \quad 0$

O $\quad 0 \quad 0 \quad 0$

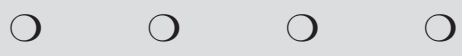

○ $\bigcirc \quad \bigcirc$

$\begin{array}{cccc}0 & 0 & 0 & 0 \\ 0 & 0 & 0 & 0 \\ 0 & 0 & 0 & 0 \\ 0 & 0 & 0 & 0 \\ 0 & 0 & 0 & 0\end{array}$


Répondez le plus honnêtement possible.

II est normal que tous les comportements énumérés dans ce questionnaire ne soient pas toujours réalisés. Répondez au questionnaire de manière à refléter votre pratique réelle.

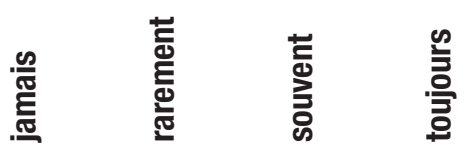

4. vérifier que les outils d'éducation et d'information en français sont adaptés à la clientèle (p. ex., contexte culturel, ethnique ou règlementaire).

5. développer des outils d'éducation et d'information qui présentent le texte en français et en anglais dans le même document.
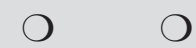

○

6. rédiger la documentation (dossiers, rapports, formulaires d'assurance, etc.) dans la langue préférée par le client

\section{Soutien et aiguillage}

Dans mon milieu de travail, il est habituel de/d'...

1. indiquer le choix langagier du client sur les formulaires utilisés lors du transfert.

$\bigcirc \quad \bigcirc \quad \bigcirc$

2. tenir une liste à jour des professionnels et des organismes dans la région ou la ville qui peuvent offrir des services en français.

3. inciter le personnel à informer les clients des possibilités d'être dirigés vers des services en français dans la région ou ailleurs.

\section{Développement professionnel continu}

Dans mon milieu de travail, des activités de formation continue sont offertes :

1. pour permettre de développer sa compétence linguistique en français.

2. sur la terminologie en français propre à notre travail.

3. sur l'offre active de services sociaux et de santé en français.
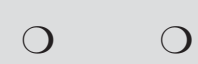

$\bigcirc$

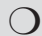

4. sur la compétence culturelle et linguistique.

5. sur les ressources facilitant l'offre de services en français.

$\bigcirc$

$\bigcirc$

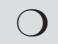

$\bigcirc$

O

O

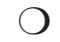

O

6. sur les enjeux et défis des communautés francophones en situation minoritaire.

7. sur les droits linguistiques.

8. des formations sont offertes en français.

$\mathrm{O}$

\section{Gestion et gouvernance}

Dans mon organisme... 
Répondez le plus honnêtement possible.

Il est normal que tous les comportements énumérés dans ce questionnaire ne soient pas toujours réalisés. Répondez au questionnaire de manière à refléter votre pratique réelle.

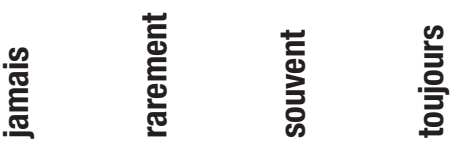

1. il y a du personnel-cadre capable de s'exprimer en français.

$\bigcirc \quad \mathrm{O}$

○

$\bigcirc$

2. il y a au moins un siège réservé à un représentant de la communauté francophone au conseil d'administration.

3. on sensibilise les intervenants aux lois, aux règlements et

$\bigcirc \quad \bigcirc$ aux politiques provinciales sur les services en français.

$\bigcirc \quad \bigcirc$

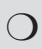

$\bigcirc$ français.

5. il est habituel de valoriser le travail fait pour offrir des services en français

$\bigcirc$

O

O

O

6. le nom officiel de l'organisme est affiché en français ou dans les deux langues officielles du Canada.

7. il y a une politique écrite sur l'offre de services en français ou dans les deux langues officielles du Canada.

\section{Obstacles}

Dans mon milieu de travail, les éléments suivants m'empêchent d'offrir des services en français autant que je le souhaiterais :

1. Attitudes du syndicat

2. Horaire trop chargé

3. Absence de leadership de l'employeur/l'organisation

$\bigcirc$

$\bigcirc$

O

$\bigcirc$

O

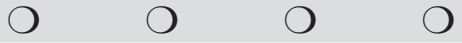

$\begin{array}{llll}0 & 0 & 0\end{array}$

$\bigcirc \quad \mathrm{O}$

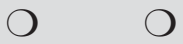

4. Pressions/préjugés de la communauté contre la prestation de services en français

5. Pressions/préjugés des collègues contre la prestation de services en français

$\bigcirc \quad \bigcirc$

$\bigcirc$

Environnement de travail ne favorise pas l'utilisation du français

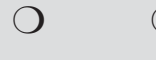

$\bigcirc \quad$

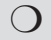

○

* 1 les passages en italiques indiquent des passages qui ont été reformulés après la première expérimentation du questionnaire. 